\title{
Obstrução arterial retiniana periférica associada com hiper-homocisteinemia: relato de caso
}

\author{
Peripheral retinal arterial obstruction associated \\ with hyperhomocysteinemia: case report
}

\author{
Alexandre Kazuo Misawa ${ }^{1}$ \\ Hisashi Suzuki² \\ Otacílio de Oliveira Maia Júnior ${ }^{3}$ \\ Maria Teresa Brizzi Chizzotti Bonanomi ${ }^{4}$ \\ Carlos Sérgio Nascimento de Melo ${ }^{5}$
}

Trabalho desenvolvido no Departamento de Oftalmologia do Hospital das Clínicas da Faculdade de Medicina da Universidade de São Paulo - USP - Setor de Retina e Vítreo - São Paulo (SP) - Brasil.

Médico Estagiário do Departamento de Oftalmologia do Hospital das Clínicas da Faculdade de Medicina da Universidade de São Paulo - USP - São Paulo (SP) Brasil.

${ }^{2}$ Professor Associado da Faculdade de Medicina da USP - São Paulo (SP) - Brasil.

3 Especialista em Retina e Vítreo do Hospital São Rafael, Fundação Monte Tabor. Professor Substituto da Faculdade de Medicina da Universidade Federal da Bahia UFBA - Salvador (BA) - Brasil.

${ }^{4}$ Médica Assistente Doutora do Departamento de Oftalmologia do Hospital das Clínicas da Faculdade de Medicina da USP - São Paulo (SP) - Brasil

5 Médico Estagiário do Departamento de Oftalmologia do Hospital das Clínicas da Faculdade de Medicina da USP - São Paulo (SP) - Brasil.

Endereço para correspondência: Otacílio de Oliveira Maia Júnior. Hospital São Rafael, Fundação Monte Tabor. Av. São Rafael, 2.142 - Salvador (BA)

CEP 41253-190

E-mail: omaiausp@uol.com.br

Recebido para publicação em 17.04.2007

Última versão recebida em 12.08.2007

Aprovação em 22.08.2007

Nota Editorial: Depois de concluída a análise do artigo sob sigilo editorial e com a anuência do Dr. Márcio Bittar Nehemy sobre a divulgação de seu nome como revisor, agradecemos sua participação neste processo.

\begin{tabular}{|l|}
\hline RESUMO \\
\hline A hiperhomocisteinemia é fator de risco para fenômenos trombo-embólicos \\
retinianos associados a quadro de oclusão vascular venosa e arterial. \\
Descrevemos um paciente com obstrução arterial retiniana periférica, sem \\
sinais de vasculite ativa, associada a proliferação de neovasos com tração \\
vítreo-retiniana e hemorragia vítrea recidivante. O alto nível sérico de \\
homocisteína decorrente de deficiência de vitamina B $_{12}$ eácido fólico, sem \\
outras alterações na cascata da coagulação, inclusive com a pesquisa do \\
fator V de Leiden, sugere que a hiper-homocisteinemia esteja diretamente \\
ligada como fator causal deste quadro clínico. Embora apresentasse PPD \\
elevado, o diagnóstico diferencial mais importante de doença de Eales foi \\
menos considerado por ser diagnóstico de exclusão. O controle do quadro \\
clínico foi feito com suplemento de vitaminas (B ${ }_{12}$ e ácido fólico) e foto- \\
coagulação retiniana periférica. A homocisteína plasmática total deve ser \\
dosada em pacientes com obstrução vascular retiniana, já que a hiper- \\
homocisteinemia é fator de risco modificável e de fácil tratamento por meio \\
de dieta ou suplementação vitamínica.
\end{tabular}

Descritores: Artéria retiniana/anormalidades; Oclusão da artéria retiniana; Homocisteína/ sangue; Vasculite retiniana/etiologia; Vasos retinianos; Relatos de casos [Tipo de publicação]

\section{INTRODUCÃO}

O primeiro relato de trombose arterial associada com hiper-homocisteinemia foi publicado por McCully em 1969, sobre duas crianças com homocistinúria hereditária, caracterizada por retardo mental, anormalidades esqueléticas, oclusões vasculares e alterações oftalmológicas: ectopia de cristalino, degeneração retiniana periférica com risco para descolamento e obstrução vascular retiniana ${ }^{(1-2)}$. Desde então, outros autores têm relacionado a hiper-homocisteinemia como fator de risco isolado e potencialmente modificável para aterosclerose e aterotrombose ${ }^{(3-5)}$.

A homocisteína é um aminoácido essencial que participa da síntese de dois outros aminoácidos, metionina e cisteína, através das vias enzimáticas da remetilação e da transulfuração, respectivamente ${ }^{(3,5-6)}$. Nesse processo metabólico, há participação das enzimas metionina sintetase, metilenotetrahidrofolato redutase e cistationina-beta-sintetase com a participação das vitaminas $\mathrm{B}_{6}, \mathrm{~B}_{12}$ e do ácido fólico. A homocisteína plasmática total em jejum varia de 5 a $15 \mu \mathrm{M} / \mathrm{L}$ no adulto normal, sendo considerada hiperhomocisteinemia grave aquela acima de $100 \mu \mathrm{M} / \mathrm{L}^{(3,5)}$. O defeito genético leva à manifestação clínica de homocistinúria ${ }^{(2)}$ e o quadro adquirido tem associação com eventos obstrutivos coronarianos e vasculares periféricos, inclusive retinianos ${ }^{(5)}$. 
Este relato descreve um paciente que apresentou hemorragia vítrea secundária à proliferação de neovasos retinianos periféricos associado à hiper-homocisteinemia.

\section{RELATO DE CASO}

EMC, 32 anos, sexo masculino, pardo, procedente de São Paulo, queixava-se de perda súbita da visão no olho direito (OD) há 2 dias, sem queixas sistêmicas. Referiu um episódio semelhante no mesmo olho há 4 anos, diagnosticado como hemorragia vítrea com resolução espontânea. Como antecedente pessoal, havia tido contato com irmão portador de tuberculose pulmonar em tratamento com esquema I (rifampicina, isoniazida e pirazinamida) há 2 meses. Ao exame, apresentava acuidade visual corrigida de conta dedos a $30 \mathrm{~cm}$ no OD e de 20/20 no olho esquerdo (OE); pressão intra-ocular de $16 \mathrm{mmHg}$ em ambos os olhos (AO), biomicroscopia e gonioscopia sem alterações em AO. A fundoscopia no OD mostrou hemorragia vítrea associada a proliferação fibrovascular e, no $\mathrm{OE}$, neovasos retinianos tracionados para o vítreo, semelhantes aos "sea-fan", na retina superior e nasal, associados a vários pontos de hiperplasia do epitélio pigmentado da retina (EPR) seguindo os vasos, mas sem sinais de vasculite ativa (Figura 1).

Os exames laboratoriais afastaram doença falciforme, evidenciando anemia macrocítica por deficiência de vitaminas $\left(\mathrm{B}_{12}\right.$ e ácido fólico), hiper-homocisteinemia e um PPD forte reator (Quadro 1). Optou-se por reposições de Vitamina $B_{12}(1000 \mathrm{U}$ IM 1x/semana por 1 mês) e ácido fólico (5 mg/dia VO por 1 mês), profilaxia para tuberculose com isoniazida por 2 meses, apesar de ter sido afastada doença ativa, conduta expectante no OD e fotocoagulação setorial com laser de argônio no OE (Figura 2). Após 1 mês do tratamento, os níveis séricos da homocis-

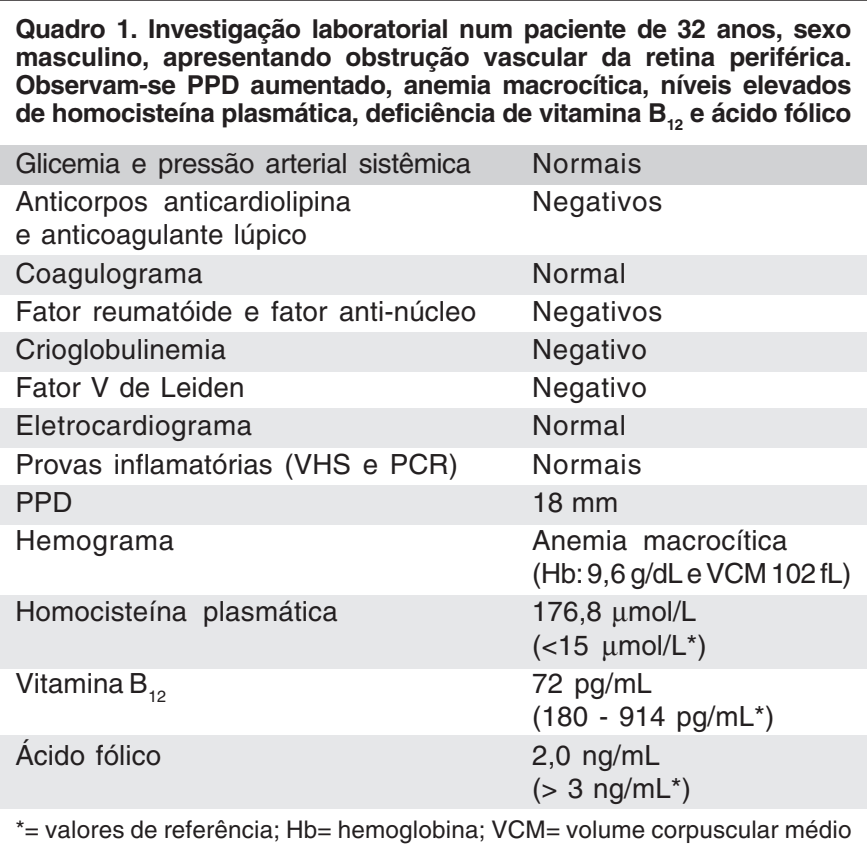
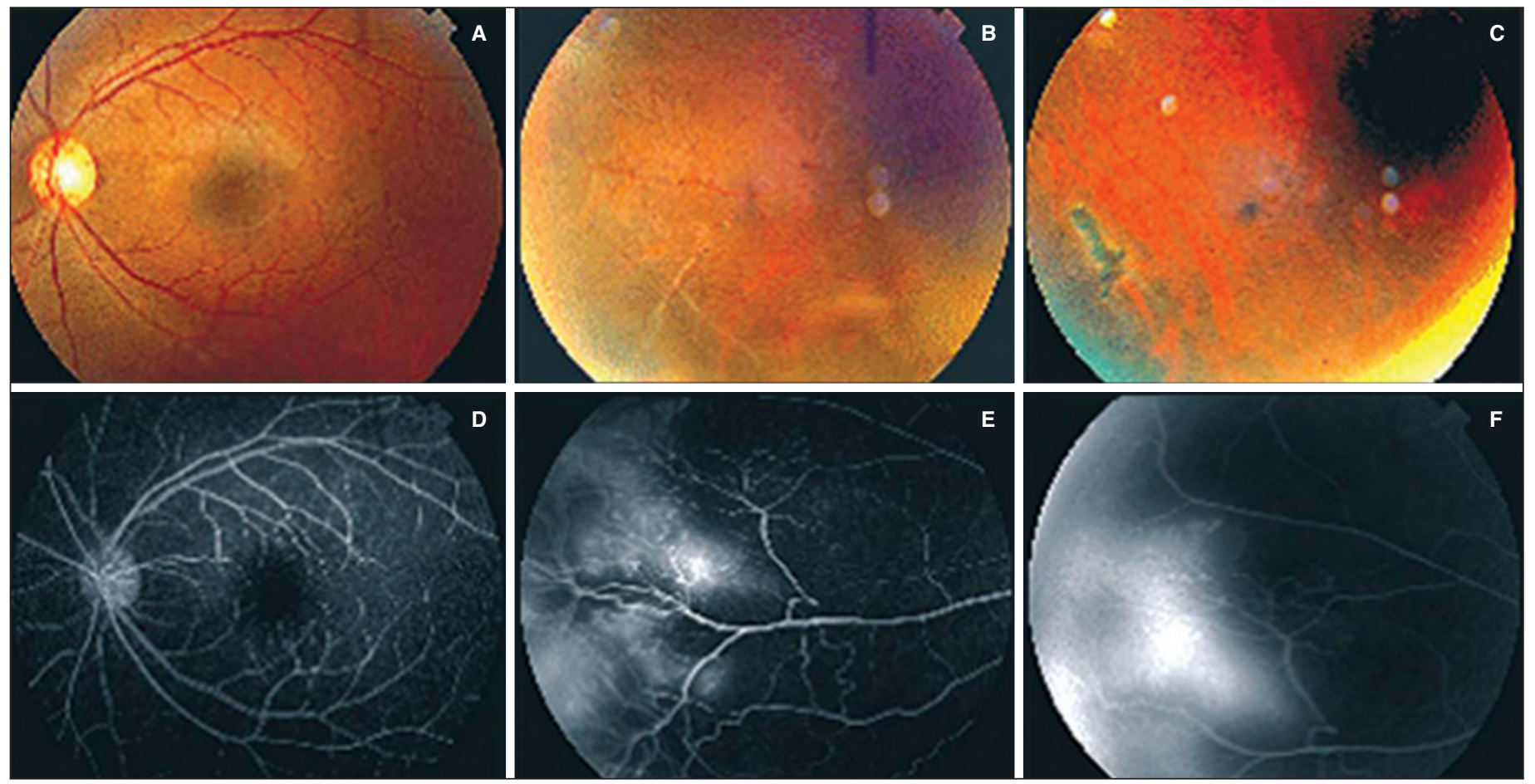

Figura 1 - Retinografia do olho esquerdo, mostrando pólo posterior sem alterações (A), tecido fibroglial com vasos "fantasmas" (B) e áreas de hiperplasia do epitélio pigmentado da retina (C) na periferia da retina. A angiofluresceinografia do olho esquerdo evidencia o pólo posterior sem alteração (D), e a presença de neovasos perfundidos na proliferação fibroglial periférica (E,F). Não foi possível obter imagem da retina do olho direito, em virtude de hemorragia vítrea. 
teína $(12 \mu \mathrm{mol} / \mathrm{L})$, vitamina $\mathrm{B}_{12}(715 \mathrm{pg} / \mathrm{mL})$ e do ácido fólico $(19,9 \mathrm{ng} / \mathrm{mL})$ foram reestabelecidos à normalidade, com melhora da anemia. Nesse período, no OD, a hemorragia vítrea já havia sido reabsorvida, apresentando áreas de hiperplasia do EPR e tração vítreo-retiniana, não sendo necessário tratamento. A neovascularização no $\mathrm{OE}$ apresentou-se estável com a laserterapia. O paciente encontra-se em acompanhamento ambulatorial há 3 anos sem recidiva do quadro oftalmológico e acuidade de 20/20 em AO.

\section{DISCUSSÃO}

A hiper-homocisteinemia pode ser secundária a um erro inato do metabolismo, resultando em elevação acentuada de homocisteína no plasma (geralmente acima de $100 \mu \mathrm{mol} / \mathrm{L}$ ) e na urina (homocistinúria). As causas mais comuns de hiper-ho- mocisteinemia são as deficiências enzimáticas e as nutricionais de ácido fólico, vitaminas $\mathrm{B}_{12}$ e $\mathrm{B}_{6}^{(3,5-6)}$. Deficiências de vitamina $B_{12}$ e do co-substrato folato podem elevar significativamente a homocisteína sérica, sendo que níveis séricos inadequados de vitaminas do complexo $\mathrm{B}$ respondem por aproximadamente $65 \%$ de todos os casos de hiper-homocisteinemia ${ }^{(7)}$

O mecanismo fisiopatológico exato pelo qual a homocisteína causa fenômenos tromboembólicos ainda é incerto, mas parece estar associado com disfunção e lesão endotelial, seguida de ativação de plaquetas e formação de trombo por diversos mecanismos: exposição da matriz subendotelial, perda da regulação vasomotora, formação de oxigênio reativo (peróxido de hidrogênio e superóxidos) e, com o estresse endoplasmático, desregulando o metabolismo lipídico gerando apoptose e inflamação das células endoteliais e promovendo formação de trombos com conseqüente obstrução vascular ${ }^{(5,7-9)}$.
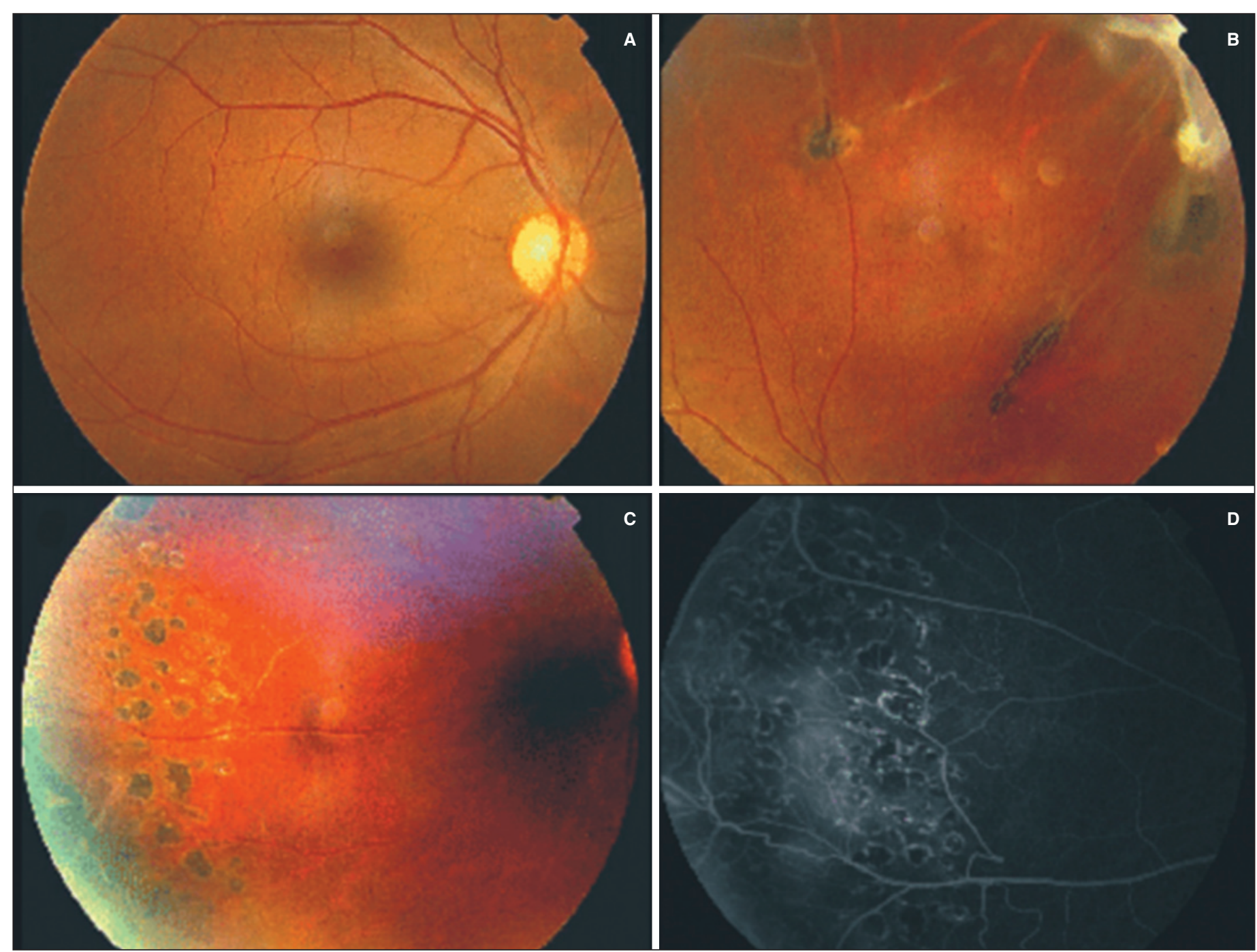

Figura 2 - Retinografia do olho direito, após a reabsorção da hemorragia vítrea. Vê-se a região macular sem alterações (A) e, na periferia, áreas de hiperplasia do epitélio pigmentado da retina, vasos "fantasmas" e traves de fibrose vítreo-retiniana (B). Retinografia (C) e angiofluoresceinografia (D) da retina periférica do olho esquerdo, mostrando as marcas coalescentes de laser da fotocoagulação setorial e a presença de neovasos pré-retinianos residuais. 
Estudos demonstram que hiper-homocisteinemia é considerada fator de risco para doença vascular retiniana, porém a deficiência congênita da enzima metileno-tetrahidrofolato redutase não está relacionada com aumento do risco do fenômeno trombótico ${ }^{(10-15)}$.

A hiper-homocisteinemia é também considerada fator de risco para obstrução de veia central da retina (OVCR) e de ramo de veia central da retina. Alguns autores identificaram, em estudo retrospectivo, a homocisteína como fator de risco para $\mathrm{OVCR}^{(4)}$. Outros estudos reforçaram esta associação ${ }^{(8,6,16)}$. Nível de homocisteína maior que $15 \mu \mathrm{mol} / \mathrm{L}$ está associado com risco três vezes maior de desenvolver doença vascular retinia$\mathrm{na}^{(11,17)}$, com obstrução venosa ou arterial, especialmente em pacientes abaixo dos 50 anos de idade ${ }^{(18-19)}$. Apesar disso, o "Blue Montains Eye Study" não encontrou associação entre hiper-homocisteinemia e a ocorrência de êmbolos nos vasos retinianos ${ }^{(9)}$.

Uma meta-análise realizada por outros autores, analisou um total de 614 pacientes e verificou que a doença vascular retiniana oclusiva estava associada com nível plasmático alto de homocisteína e baixo de ácido fólico, sem associação com os níveis séricos de vitamina $\mathrm{B}_{12}$ e $\mathrm{B}_{6}{ }^{(5)}$. Apesar disso, o uso de vitamina $\mathrm{B}_{12}$ reduziu níveis de homocisteína plasmática; sendo, assim, aconselhável como terapia coadjuvante. A oclusão dos vasos periféricos da retina é relatada raramente em associação com a trombofilia secundária a hiper-homocisteinemia. Um quadro clínico semelhante ao apresentado foi descrito na mutação do fator $\mathrm{V}$ (fator de Leiden) num paciente com hemorragia vítrea e neovascularização periférica da retina temporal ${ }^{(15)}$.

No presente relato, o diagnóstico diferencial deve ser feito com doença de Eales, tanto pela hemorragia vítrea como pela oclusão vascular e cicatrizes coriorretinianas. No entanto, a doença de Eales não tem etiologia definida, sendo descrita em homens jovens, saudáveis e mais comumente encontrada no subcontinente indiano. Caracteriza-se por vasculite retiniana periférica primária, mais comumente como periflebite que evolui para estágio isquêmico com proliferação de neovasos e conseqüente hemorragia vítrea. A etiologia ainda está indefinida, acreditando-se que seja multifatorial (imunológica, parasitária e molecular), com uma associação com partículas do Micobacterium tuberculosis, detectadas no humor vítreo, sendo seu diagnóstico presuntivo ${ }^{(20-21)}$.

Portanto, relatou-se um paciente com obstrução arterial retiniana periférica associada a proliferação de neovasos com tração vítreo-retiniana periférica e hemorragia vítrea recidivante, sem sinais de vasculite ativa. $\mathrm{O}$ alto nível sérico de homocisteína, decorrente de deficiência de vitamina $\mathrm{B}_{12} \mathrm{e}$ ácido fólico, sem outras alterações que pudessem ser detectadas na cascata da coagulação, sugere que a hiper-homocisteinemia esteja associada a este quadro clínico. Apesar de o paciente apresentar PPD elevado, ter tido contato com o irmão portador tuberculose e apresentar zonas de hiperplasia do EPR ao longo de alguns vasos retinianos, é pouco provável que a hipersensibilidade tuberculínica tenha relação com este quadro retiniano atípico. Apenas a reposição nutricional de vitaminas e o tratamento com laser de argônio estabilizaram o quadro clínico, sem necessidade do uso de antiinflamatórios. A homocisteína plasmática total deve ser dosada em pacientes com obstrução vascular retiniana, por ser um fator de risco modificável e de fácil tratamento. Uma vez diagnosticada a hiper-homocisteinemia, impõe-se a pesquisa de doenças de base associadas para que se possa obter uma melhor eficácia terapêutica.

\section{ABSTRACT}

Hyperhomocysteinemia is a risk factor for thromboembolic events of the retina associated with vascular venous or arterial occlusion. We describe a patient with occlusion of the peripheral arteriolar network without active vasculitis, associated with neovascular proliferation, peripheral vitreous-retinal traction and relapsing vitreous hemorrhage. The high serum homocysteine level resulting from vitamin $\mathrm{B}_{12}$ and folic acid deficiency, without further changes in the coagulation cascade including the test for Leiden's Factor V, indicates hyperhomocysteinemia as a direct causal factor in this clinical condition. Despite a high PPD, Eales Disease, a major differential diagnosis, was not fully considered, since it is established by exclusion. The patient was treated with photocoagulation and vitamin supplements and the condition was successfully controlled. Patients with retinal vascular obstruction should have their total plasma homocysteine levels measured, since this modifiable risk factor can be easily treated with dietary approaches including vitamin supplementation.

Keywords: Retinal artery/abnormalities; Retinal artery occlusion; Homocysteine/blood; Retinal vasculitis/etiologia; Retinal vessels; Case reports [Publication type]

\section{REFERÊNCIAS}

1. McCully KS. Vascular pathology of homocysteinemia: implications for the pathogenesis of arteriosclerosis. Am J Pathol. 1969;56(1):111-28.

2. Vd Berg W, Verbraak FD, Bos PJ. Homocystinuria presenting as central retina artery occlusion and longstanding thromboembolic disease. Br J Ophthalmol. 1990;74(11):696-7.

3. Welch GN, Loscalzo J. Homocysteine and atherothrombosis. N Engl J Med. 1998;338(15):1042-50.

4. Vine AK. Hyperhomocysteinemia: a risk factor for central retinal vein occlusion Am J Ophthtalmol. 2000;129(5):640-4. Comment in: Am J Ophthalmol. 2001; 131(2):290-1.

5. Cahill MT, Stinnett SS, Fekrat S. Meta-analysis of plasma homocysteine, serum folate, serum vitamin B12, and thermolabile MTHFR genotype as risk factor for retinal vascular occlusive disease. Am J Ophthalmol. 2003;136(6):1136-50.

6. Yildirim C, Yaylali V, Tatlipinar S, Kaptanoglu B, Akpinar S. Hyperhomocysteinemia: a risk factor for retinal vein occlusion. Ophthalmologica. 2004; 218(2):102-6.

7. Selhub J, Jacques PF, Wilson PW, Rush D, Rosenberg IH. Vitamin status and intake as primary determinants of homocysteinemia in an elderly population. JAMA. 1993;270(22):2693-8. Comment in: JAMA. 1993;270(22):2726-7.

8. Fegan CD. Central retinal vein occlusion and thrombophilia. Eye. 2002; 16(1):98-106.

9. Chua B, Kifley A, Wong TY, Mitchell P. Homocysteine and retinal emboli: The Blue Mountains Eye Study. Am J Ophthalmol. 2006;142(2):322-4. 
10. Chak M, Wallace GR, Graham EM, Stanford MR. Thrombophilia: genetic polymorphisms and their association with retinal vascular occlusive disease. $\mathrm{Br}$ J Ophthalmol. 2001;85(7):883-6.

11. Weger M, Stanger O, Deutschmann H, Leitner FJ, Renner W, Schmut O, et al. The role of hyperhomocysteinemia and methylenetetrahydrofolate reductase (MTHFR) C677T mutation in patients with retinal artery occlusion. Am. J Ophthalmol. 2002;134(1):57-61.

12. Di Crecchio L, Parodi MB, Sanguinetti G, Iacono P, Ravalico G. Hyperhomocysteinemia and the methylenetetrahydrofolate reductase $677 \mathrm{C}-\mathrm{T}$ mutation in patients under 50 years of age affected by central retinal vein occlusion. Ophthalmology. 2004;111(5):940-5.

13. Weger M, Stanger O, Deutschmann H, Temmel W, Renner W, Schmut O, et al. Hyperhomocyst(e)inemia and MTHFR C677T genotypes in patients with central retinal vein occlusion. Graefe's Arch Clin Exp Ophthalmol. 2002; 240(4):286-90.

14. Weger M, Stanger O, Deutschmann H, Temmel W, Renner W, Schmut O, et al. Hyperhomocyst(e)inemia, but not MTHFR C677T mutation, as a risk factor in brach retinal vein occlusion. Ophthalmology. 2002;109(6):1105-9.
15. Eller AW, Bontempo FA, Faruki H, Hassett AC. Peripheral retinal neovascularization (Eales Disease) associated with the factor V Leiden mutation. Am J Ophthalmol. 1998;126(1):146-9.

16. Brown BA, Marx JL, Ward TP, Hollifield RD, Dick JS, Brozetti JJ, et al. Homocysteine: a risk factor for retinal venous occlusive disease. Ophthalmology. 2002;109(2):287-90.

17. Chua B, Kifley A, Wong TY, Mitchell P. Homocysteine and retinal vein occlusion: a population-based study. Am J Ophthalmol. 2005; 139(1):181-2.

18. Wenzler EM, Rademakers AJ, Boers GH, Cruysberg JR, Webers CA, Deutman AF. Hyperhomocysteinemia in retinal artery and retinal vein occlusion. Am J Ophthalmol. 1993;115(2):162-7.

19. Narin F, Narin N, Halici C, Oner AO, Dogan H, Karakukcu M. Plasma homocysteine and retinal artery occlusive disease: a case control study. Ann Saudi Med. 2004;24(3):186-8.

20. Biswas J, Sharma T, Gopal L, Madhavan HN, Sulochana KN, Ramakrishnan S. Eales Disease - An Update. Surv Ophthalmol. 2002;47(3):197-214.

21. Barbosa FAC, Suzuki H. Doença de Eales. In: Abujamra S. Retina e vitreo: clínica e cirúrgica. São Paulo: Roca; 2000. p.546-50. 